\title{
ON CONVEXITY OF LEVEL CURVES OF HARMONIC FUNCTIONS IN THE HYPERBOLIC PLANE
}

\author{
M. PAPADIMITRAKIS
}

(Communicated by Clifford J. Earle, Jr.)

\begin{abstract}
We prove that if two level curves of a harmonic function are convex in the hyperbolic disc then all intermediate level curves are also convex.
\end{abstract}

\section{INTRODUCTION}

Proofs of the following theorem and related results can be found in [1-6] and $[8-11]$.

Theorem. Suppose that $B$ and $U$ are two nonempty bounded convex sets in $\mathbf{R}^{n}, B$ is closed, $U$ is open, and $B \subseteq U$. Also suppose that $u$ is a real-valued function harmonic in $U \backslash B$ and continuous in $\overline{U \backslash B}$ such that

$$
\begin{array}{ll}
u \equiv 1 & \text { on } \partial B \\
u \equiv 0 & \text { on } \partial U .
\end{array}
$$

Then the set $\{x: u(x) \geq \lambda\} \cup B$ is convex for every $\lambda, 0<\lambda<1$.

The purpose of this paper is to give a proof of the same result but in the context of hyperbolic geometry in the Poincaré plane.

Assume that $\mathbf{C}$ is the complex plane, $D=\{z \in \mathbf{C}:|z|<1\}$ and that $D$ is equipped with the Poincaré metric:

$$
d s=\frac{2|d z|}{1-|z|^{2}} .
$$

This metric defines a Laplace-Beltrami operator (see [7]) $\Delta_{s}$, which is related to the ordinary Laplacian $\Delta$ by

$$
\Delta_{s} f(z)=\left(\frac{1-|z|^{2}}{2}\right)^{2} \Delta f(z) .
$$

A function $u$ defined on an open subset of $D$ is called harmonic if $\Delta_{s} u \equiv 0$. Hence the harmonic functions in the hyperbolic geometry of $D$ coincide with the ordinary harmonic functions.

Received by the editors July 9, 1990.

1980 Mathematics Subject Classification (1985 Revision). Primary 31C05, 52A55. 
It is also known that the isometries of $D$ are the Möbius transformations,

$$
T(z)=\lambda \cdot \frac{z-a}{1-\bar{a} z}, \quad|a|<1, \quad|\lambda|=1,
$$

and that the geodesics are arcs of circles orthogonal to $\partial D$. Hence every two points of $D$ can be joined by a unique geodesic. A subset $A$ of $D$ is called convex if for every two points of $A$ the whole geodesic segment joining these two points is in $A$.

\section{THE RESULT}

Theorem. Suppose that $D$ is the unit disc with the hyperbolic geometry, and $B$ and $U$ are two nonempty convex subsets of $D$ such that $B$ is closed, $U$ is open, $B \subseteq U, \bar{U} \subseteq D$. Also suppose that $u$ is a real-valued function harmonic in $U \backslash B$ and continuous on $\overline{U \backslash B}$ with

$$
\begin{array}{ll}
u \equiv 1 & \text { on } \partial B \\
u \equiv 0 & \text { on } \partial U .
\end{array}
$$

Then the set $\{z: u(z) \geq \lambda\} \cup B$ is convex for every $\lambda, 0<\lambda<1$.

Proof. We can easily reduce the general case to the case where $\partial U$ and $\partial B$ are smooth. Next we consider a conformal mapping $\phi$ from some ring domain:

$$
D(R, 1)=\{z \in \mathbf{C}: R<|z|<1\}
$$

to $U \backslash B$. Then

$$
\begin{gathered}
\phi: C_{1}=\{|z|=1\} \rightarrow \partial U, \\
\phi: C_{R}=\{|z|=R\} \rightarrow \partial B
\end{gathered}
$$

are homeomorphisms.

Since $u$ is harmonic, $\nu=u \circ \phi$ is harmonic in $D(R, 1)$ with

$$
\begin{array}{ll}
\nu \equiv 0 & \text { on } C_{1}, \\
\nu \equiv 1 & \text { on } C_{R} .
\end{array}
$$

This implies that $\nu(z)=\log |z| / \log R$ and the level curves of $\nu$ are concentric circles. So we need to prove that

$$
\phi\left(C_{r}\right) \text { is a convex curve (in the Poincaré metric) }
$$

for every $r, R<r<1$.

It is trivial to prove that, in the Euclidean metric of $\mathbf{R}^{2}, \phi\left(C_{r}\right)$ is convex if and only if

$$
\operatorname{Re}\left\{\zeta \cdot \frac{\phi^{\prime \prime}(\zeta)}{\phi^{\prime}(\zeta)}+1\right\} \geq 0, \quad \forall \zeta \in C_{r}
$$

Since Möbius transformations preserve convexity (in hyperbolic geometry), if we want to study convexity of $\phi\left(C_{r}\right)$ at some point $\phi(\zeta)$, we can equivalently study convexity of $T\left(\phi\left(C_{r}\right)\right)$ at $T(\phi(\zeta))$ where $T$ is any Möbius transformation.

We may use $T(z)=(z-\phi(\zeta)) /(1-\overline{\phi(\zeta)} \cdot z)$, which maps $\phi(\zeta)$ to 0 . But at 0 the hyperbolic and the Euclidean geometry coincide, hence the condition for convexity of $T\left(\phi\left(C_{r}\right)\right)$ at 0 is

$$
\operatorname{Re}\left\{\zeta \cdot \frac{(T \circ \phi)^{\prime \prime}(\zeta)}{(T \circ \phi)^{\prime}(\zeta)}+1\right\} \geq 0
$$


which after elementary calculations becomes

$$
\operatorname{Re}\left\{\zeta \frac{\phi^{\prime \prime}(\zeta)}{\phi^{\prime}(\zeta)}+2 \zeta \frac{\overline{\phi(\zeta)} \cdot \phi^{\prime}(\zeta)}{1-|\phi(\zeta)|^{2}}+1\right\} \geq 0
$$

Let us denote the left side by $p(\zeta)$. The problem reduces to showing that if $p \geq 0$ on $C_{1}$ and on $C_{R}$ then $p \geq 0$ on $C_{r}$ for every $r, R<r<1$. (Note that in the Euclidean geometry the middle term is missing, hence $p$ is harmonic and the result is a trivial application of the maximum principle for $p$.)

Now, let $\Delta$ be the Euclidean Laplacian.

$$
\begin{aligned}
\Delta p & =\operatorname{Re} \Delta\left[\frac{2 \zeta \overline{\phi(\zeta)} \cdot \phi^{\prime}(\zeta)}{1-|\phi(\zeta)|^{2}}\right]=\operatorname{Re} 4 \cdot \frac{\partial^{2}}{\partial \zeta \partial \bar{\zeta}}\left[\frac{2 \cdot \zeta \cdot \bar{\phi} \cdot \phi^{\prime}}{1-|\phi|^{2}}\right] \\
& =8 \operatorname{Re} \frac{\partial}{\partial \zeta}\left[\zeta \cdot \phi^{\prime} \cdot \frac{\partial}{\partial \bar{\zeta}}\left(\frac{\bar{\phi}}{1-\phi \cdot \bar{\phi}}\right)\right]=8 \operatorname{Re} \frac{\partial}{\partial \zeta}\left[\zeta \cdot \frac{\left|\phi^{\prime}\right|^{2}}{\left(1-|\phi|^{2}\right)^{2}}\right] \\
& =8 \frac{\left|\phi^{\prime}\right|^{2}}{\left(1-|\phi|^{2}\right)^{2}} \cdot \operatorname{Re}\left[1+\zeta \cdot \frac{\phi^{\prime \prime}}{\phi^{\prime}}+2 \zeta \cdot \frac{\bar{\phi} \cdot \phi^{\prime}}{1-|\phi|^{2}}\right]
\end{aligned}
$$

Hence

$$
\Delta p=8 \cdot \frac{\left|\phi^{\prime}\right|^{2}}{\left(1-|\phi|^{2}\right)^{2}} \cdot p=k \cdot p,
$$

where $k$ is a positive function.

Now suppose that $p$ is negative at some interior point of $D(R, 1)$. Then $p$ has a negative minimum at some point $\zeta$ :

$$
\min p=p(\zeta)<0, \quad \zeta \in D(R, 1) .
$$

But then $\Delta p(\zeta) \geq 0$, contradicting (1). Hence $p \geq 0$ in $D(R, 1)$ and the proof is complete.

It might be interesting to see what happens in the hyperbolic geometry in more than two dimensions, and perhaps one might be able to characterize those Riemannian manifolds for which the same theorem is true. In [11] a Riemannian metric for $\mathbf{R}^{2}$ is constructed for which the theorem fails.

\section{REFERENCES}

1. L. A. Caffarelli and A. Friedman, Convexity of solutions of semilinear elliptic equations, Duke Math. J. 52 (1985), 431-456.

2. L. A. Caffarelli and J. Spruck, Convexity properties of solutions to some classical variational problems, Comm. Partial Differential Equations 7 (1982), 1337-1379.

3. R. Gabriel, An extended principle of the maximum for harmonic functions in 3 dimensions, J. London Math. Soc. (2) 30 (1955), 388-401.

4. _ A result concerning convex level surfaces of 3-dimensional harmonic functions, $\mathbf{J}$. London Math. Soc. (2) 32 (1957), 286-294.

5. ___ Further results concerning the level surfaces of the Green's function for a 3-dimensional convex domain, II, J. London Math. Soc. (2) 32 (1957), 303-306.

6. J. J. Gergen, Note on the Green function of a star-shaped three dimensional region, Amer. J. Math. 53 (1931), 746-752.

7. S. Helgason, Differential geometry and symmetric spaces, Academic Press, New York, 1962. 
8. N. Korevaar, Convex solutions to nonlinear elliptic and parabolic boundary value problems, Indiana Univ. Math. J. 32 (1983), 603-614.

9. N. Korevaar and J. L. Lewis, Convex solutions of certain elliptic equations have constant rank Hessians, Arch. Rational. Mech. Anal. 97 (1987), 19-32.

10. J. L. Lewis, Capacitary functions in convex rings, Arch. Rational Mech. Anal. 66 (1977), 201-224.

11. J. P. Rosay and W. Rudin, A maximum principle for sums of subharmonic functions and the convexity of level sets, Michigan Math. J. 36 (1989), 95-111.

Department of Mathematics, University of Wisconsin, Madison, Wisconsin 53706 4899

Current address: Department of Mathematics, Washington University, St. Louis, Missouri 63130- 